\title{
City sustainability: a transport perspective - a journey continues
}

\author{
K. Doust ${ }^{1,2}$, C. Wang ${ }^{1} \&$ T. Doust ${ }^{2}$ \\ ${ }^{I}$ Research Centre for Integrated Transport Innovation (rCITI), \\ University of NSW, Australia \\ ${ }^{2}$ Windana Research Pty Ltd, Australia
}

\begin{abstract}
The paper explores the challenge of how to maximise sustainability while providing for citywide accessibility to opportunities. Beginning with the historical context to the concept of sustainable accessibility, the paper discusses the potential in cities for sustainable trunk transport networks. The trend in many cities to add orbital links to create a web like spatial structure to the transport system is examined. By applying evidence based assessment techniques to suitable case study cities, a goal of this research is to contribute an understanding in how achievable the concept of sustainable citywide accessibility is.

By exploring the city structures in key world cities, the research identifies cities consistent with web like spatial layouts of trunk transport corridors or with the potential to be structured with this form. Initial case studies are drawn from Sydney where suitable data is available. Evidence based sustainability metrics are derived, utilising a methodology first introduced in 2008 and refined for the current research program.

The paper briefly overviews a number of cities that have begun with a radial transport structure but transformed into a web like structure using orbital links. Based on an initial assessment of Sydney, the paper makes initial conclusions on how effective web like spatial transport structures are for sustainable citywide accessibility. The paper also foreshadows further work on orbital public transport links and introduces the opportunity and challenges involved in reengineering trunk corridors to multimodal systems. Conclusions outline continuing work needed and need for collaboration with other researchers. Ultimately, the purpose is to generate a typology of urban and transport spatial form that together with a
\end{abstract}


practical assessment methodology gives city planners additional tools for making informed sustainability decisions.

Keywords: sustainability, accessibility, city planning, transport, methodology, orbital, ring roads.

\section{Introduction}

This paper builds upon earlier observations in 2004, revisiting the efforts to achieve sustainability by focussing only on keeping trips localised as far as possible. While agreeing with this effort, the paper raises again the thinking that citywide accessibility is also required and effort should go into achieving trunk corridors that enable sustainable citywide accessibility to be achieved.

The concept of web transport structures is discussed and a sustainability assessment methodology suited to giving an evidence based view on their performance is introduced. Initial case study work is presented and discussed giving indications of the potential for this work to contribute to reshaping the structure and mode mix of trunk transport corridors.

The need for collaboration amongst researchers and city planning agencies is one of the key conclusions to further build upon this work to give a useful operational tool and a typology of transport structures and their performance, for guiding transport policy decision making.

\section{The challenge of maximising the 3 pillars of sustainability}

\subsection{Present policy approach to sustainability: Sustainability Step 1}

Beginning in the 1990s, much work has been done in restructuring urban form to achieve good local accessibility between residential and opportunities. Much has been achieved by new urbanism approaches bringing land use closer together. In many cities these measures are beginning to provide good local accessibility with more people having local opportunities. As residents take up these opportunities, there is beginning to be a shift from high VKT car trips, to more localised non motorised or public transport based trips.

Good local accessibility with increased sustainability is achievable in this way. However, while the urban planning policies in new urbanism provide the opportunity to live and work locally, not all people take the opportunity up. Manning [4] observed that the expectation that resident workers would take up local jobs when they are provided near where they live, was not a definite outcome. Jobs close to home were often overlooked for better opportunities further away. Using the 1971 census data for Sydney Australia, Manning observed that the Campbelltown job take up rates by local residents were as low as $25 \%$ in 1971 , while opportunities were available for $61 \%$ to work locally. Take up rates in Penrith were $32 \%$ in 1971 , with opportunities for $95 \%$ to work locally. This effect is still a reality today. Factors influencing this include an attachment to a job when either the person shifts residence, or employment shifts location, or match of skills to a more distant job. 


\subsection{An expanded approach to sustainability: Sustainability Step 2}

In a 2004 paper (Doust [2]), the question was posed: "is there opportunity for sustainable accessibility citywide, not only locally?" If it is achievable, it will depend on sustainable local accessibility coupled with sustainable trunk transport.

Clues to sustainable trunk transport come from a seemingly unlikely place; the network of motorway corridors. In cities such as Sydney and Melbourne middle to outer suburb orbital ring roads are being added to radial motorways, providing citywide accessibility between local areas. In Melbourne, these corridors have induced industries to relocate into the middle suburb areas in close proximity to the ring road.

This citywide web of trunk corridors is a structure that provides a citywide web of accessibility. The application of the corridors for road transport alone however, does not provide for a sustainable system or make the best use of the corridor's capacity.

Sustainability Step 2 is taking this citywide web structure of trunk corridors and adjusting the web from multiroad to multimode.

\section{Concept of sustainable accessibility}

Social equity, one of the three pillars of sustainability, has a relationship to accessibility. A measure based on residential accessibility to jobs or other opportunities provides a measure of the physical spatial equity for the people living in that location.

A key connection between another sustainability pillar, that of environmental sustainability, and accessibility is that appropriate levels of both are needed, with the idealised target being $100 \%$ of good performance in both measures. In an original concept, a city's sustainability performance in relation to the goal can be analytically quantified and simply visualised in plots on what has been called "environmental sustainability - accessibility space". The concept enables both a quantifiable measure and a visual representation of the mutual performance.

\section{Methodology}

\subsection{Desk top analysis of spatial transport structures of selected cities}

Sustainability Step 2 is believed to hinge on getting an effective multimodal trunk web structure working in cities. For cities that have grown with a radial transport structure, the transformative new structure is conceptually illustrated in Figure 1.

The spatial connectivity to other trunk corridors develops the type of web structure that provides a big step in accessibility for the population of a city. The orbital motorway principle invites us to develop a multimodal trunk web structure by overlaying an orbital public transport corridor over the top of the network of radial motorways and rail lines. 


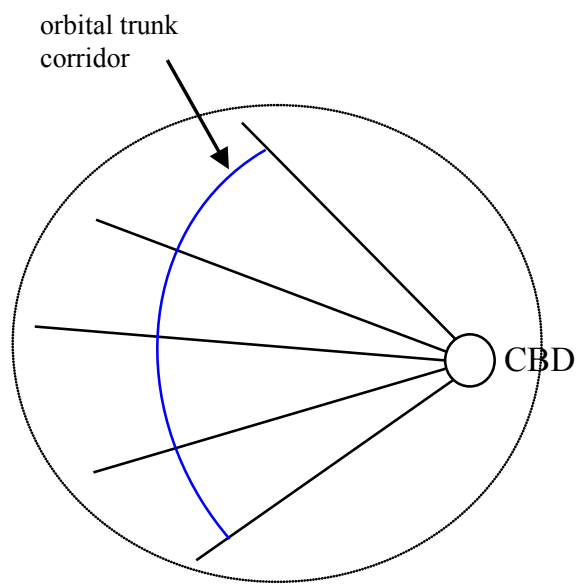

Figure 1: Creating a transport web structure (Doust [2]).

A cross section of different classifications and locations of world cities were examined using simple data sources such as google maps, wikipedia and journals, to identify cities with both a radial and an orbital road structure.

\subsection{Sustainability assessment methodology}

The strategic scan methodology outlined in Black et al. [1], based on traditional transport modelling methods, enables the metrics of sustainability to be developed from source data with traceable quantifiable steps.

For the assessment of the sustainability effectiveness of the spatial structures, a measure of $\mathrm{CO} 2$ equivalent (CO2-e) has been used. Accessibility measures use transport impedance together with employment. For the purpose of the case study testing, the socio-economic differences across Sydney and Melbourne are not applied, but could provide a further enhancement of the strategic scan.

The detailed steps in developing the metrics through derived data are shown in Doust [3]. The multiple shortest path matrix (MSPM) for census years before and after the addition of orbital road infrastructure, provides input to calculation of the transport impedance function which in turn is input to the accessibility measure (Figure 2).

\subsubsection{Operational measure of environmental sustainability}

The environmental sustainability measure for car based trips is calculated from this CO2-e unit rate data in the following manner.

The trip lengths from the MSPM for distance and the number of trips between each origin - destination $\mathrm{TZ}$ pair are multiplied providing person kilometres travelled. When coupled with car occupancy rates, this produces vehicle kilometres travelled (VKT):

$$
V K T i j=Q i j \operatorname{dij} / O c c
$$




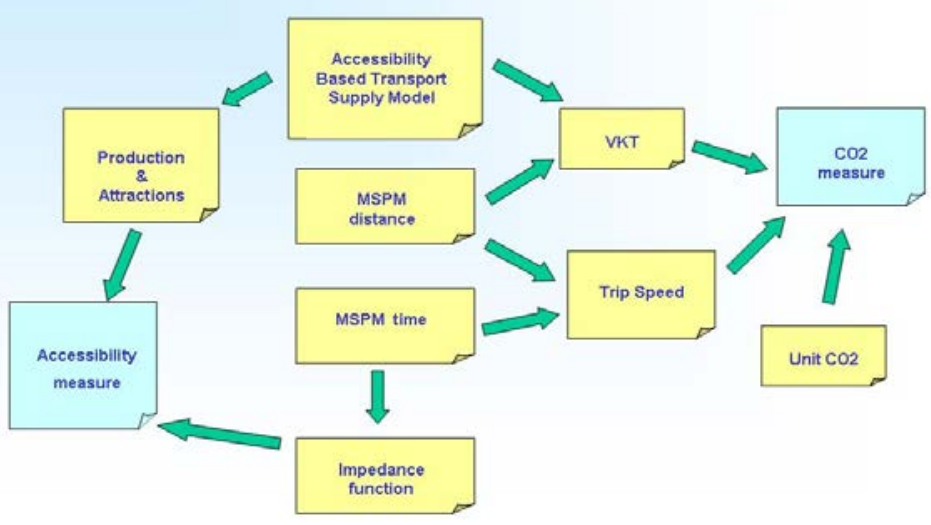

Figure 2: Accessibility measure and environmental sustainability measure, Doust [3].

where: Qij =trip frequency between TZi and TZj;

$\operatorname{dij}=$ shortest path distance between $\mathrm{TZi}$ and $\mathrm{TZj}$;

Occ $=$ ratio persons per car.

Similarly, the MSPM for time when divided into MSPM for distance provides an estimate of average speed between each origin - destination TZ pair. This is able to be coupled with an average $\mathrm{CO} 2$-e emissions index per speed of car trip, Doust [3] and the number of car trips between travel zones to give a measure of CO2-e emissions per car trip kilometre per travel zone pair. where

$$
\text { CO2-e ij/car trip/km }=S i j E
$$

An improvement in environmental sustainability occurs with a decrease in $\mathrm{CO} 2$-e emissions. For this analysis, the measure selected to plot in environmental sustainability space is:

$$
\text { Total CO2-e } i j=\text { VKTij SijE }
$$

where: $\mathrm{Sij}=$ shortest path ave speed between $\mathrm{TZi}$ and $\mathrm{TZj}$;

$\mathrm{E}=$ ratio of CO2-e per average trip speed $/$ car trip $/ \mathrm{km}$.

\subsubsection{Operational measure of accessibility}

The accessibility measure for each travel zone to employment opportunities at another travel zone is derived from the following general form of accessibility measure:

$$
H_{i}=\sum D_{J} f n\left(C_{i J}\right)
$$

where: $\mathrm{Hi}=$ accessibility measure of $\mathrm{TZi}$; 
$\mathrm{DJ}=$ potential opportunity at $\mathrm{TZj}$ measured as the total attractions from all origin zones to $\mathrm{TZj}$; $\mathrm{fn}(\mathrm{CiJ})=$ travel impedance function.

To enable comparison of each paired origin and destination zone, the operational accessibility measure is formulated as shown below with the simplest form for impedance:

$$
H_{i J}=D_{J} C_{i J}
$$

where: Hij= accessibility measure for the zonal pair TZi to TZj;

$\mathrm{Dj}=$ potential opportunity at $\mathrm{TZj}$ measured as the total attractions from all origin zones to $\mathrm{TZj}$;

$\mathrm{Cij}=$ travel impedance measured as travel time between the two zone pairs.

\section{Results}

\subsection{Orbital additions in key world cities with radial structures}

Of the cities examined it was observed that there had been an expansion in the number of cities adding orbital road links over the past 10 years.

The cities listed are typical of the addition of one or more orbitals which have served to integrate the existing radial structures into a web like structure, increasing the accessibility across the whole city. Research into the extent of how many of these orbitals have included public transport is currently progressing and will be reported in an extended version of this paper in coming months.

\subsection{Assessment of sustainability effectiveness of web like spatial structures through orbital links}

This research is focusing the case study assessment on what is commonly referred to in large cities with spatially dispersed land use as middle and outer ring communities. It is these areas that are believed to benefit most from accessibility enhancements coming from adding orbital transport corridors. This is on the basis that the web effect gains in inner ring areas have already been made through metro style public transport or because the web effect gains are less closer to the radial trunk corridors central node. However, it is also likely that the inner ring areas will also benefit from the reduction in trips that are transiting through the ring from and to middle and outer ring origins and destinations.

An initial case study analysis of a middle/outer ring orbital motor way addition in Sydney in 2004 using census data from 2001 and 2006 is currently in progress. Initial results using the 2001 data with and without the motorway has yielded the following partial analysis. The work still in progress is repeating the analysis using the 2006 data with and without the motorway, from which a comparison will be made to the 2001 results and conclusions reached on the effective change in both accessibility and carbon emissions. 


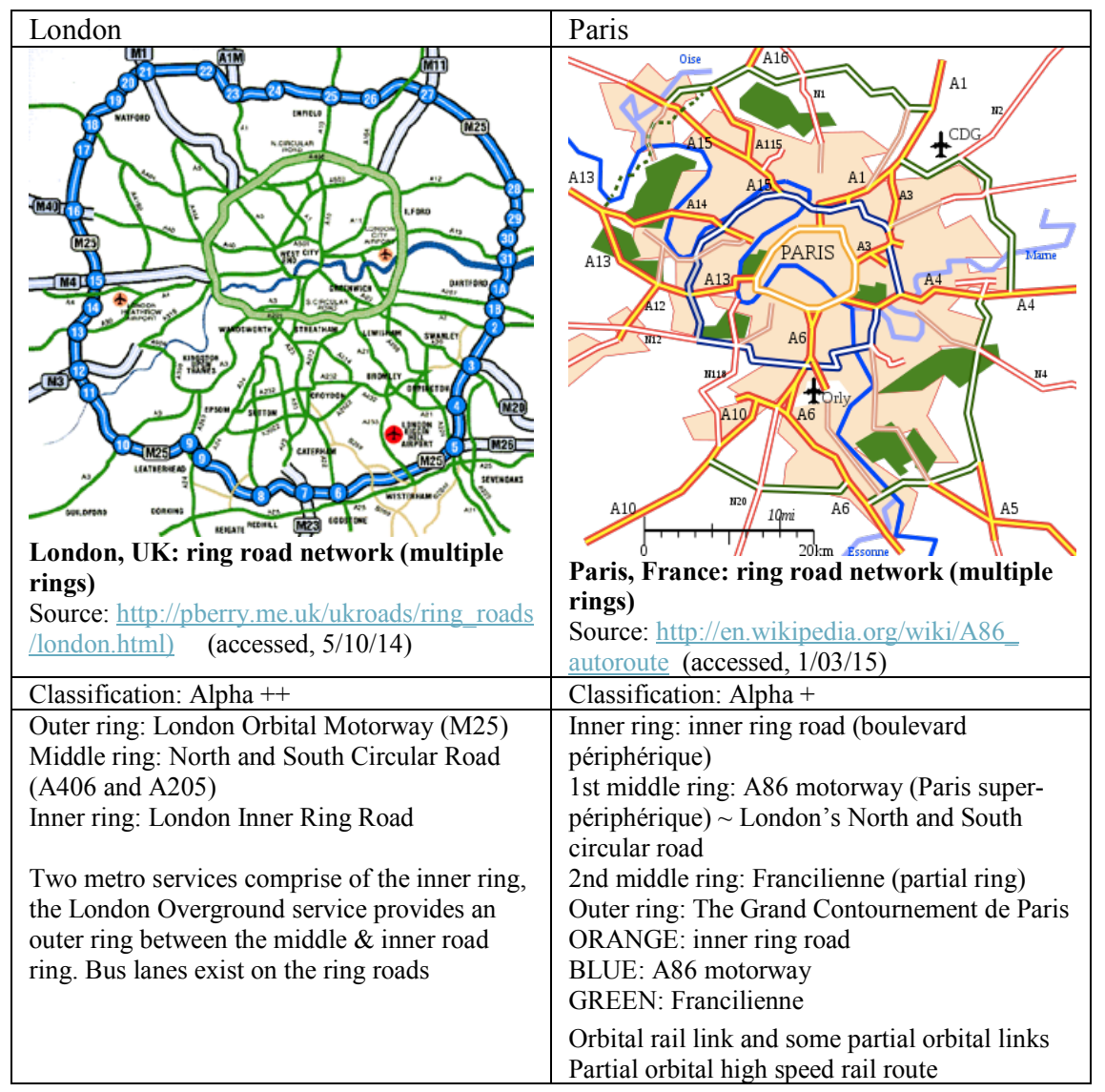

Figure 3: European cities.

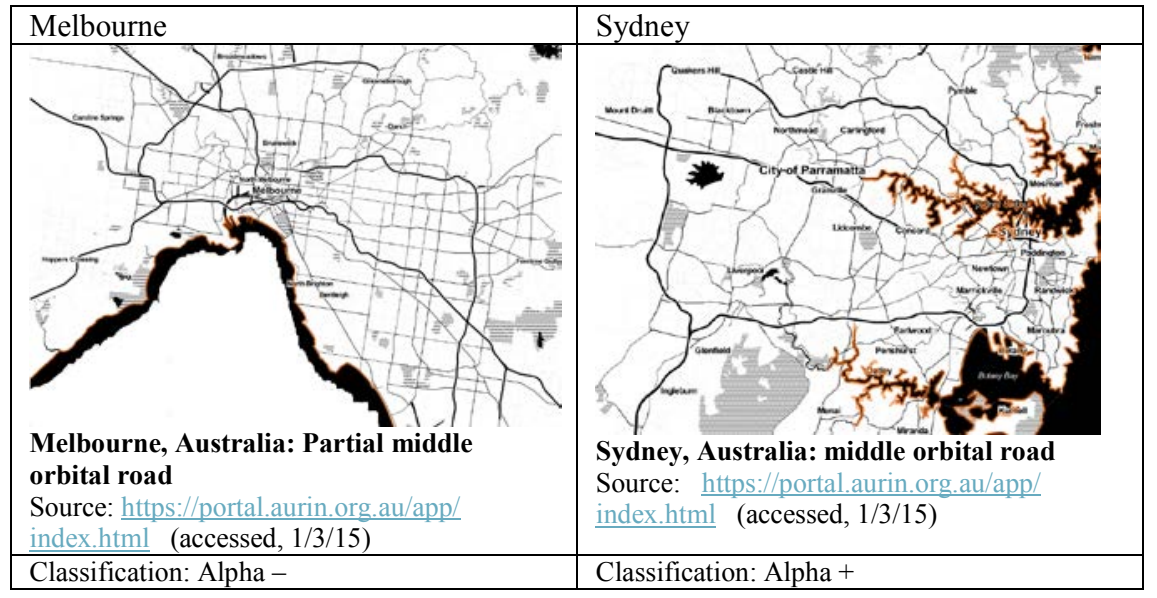

Figure 4: Australian cities. 
Figure 5 shows the absolute performance both with the M7 orbital \& without the M7 orbital. Figure 6 shows the performance with the M7 relative to the network without the M7. These preliminary results show an increase in both accessibility and a reduction in carbon emissions due to addition of the M7 orbital link for a number of travel zone origin and destination pairs with no indications of a worsening of performance for any pair. To know where these pairs are, the data above would need to be tagged with the level of improvement and replotted in GIS space to give geographical locations of each pair with a colour, coded to indicate the level of improvement.

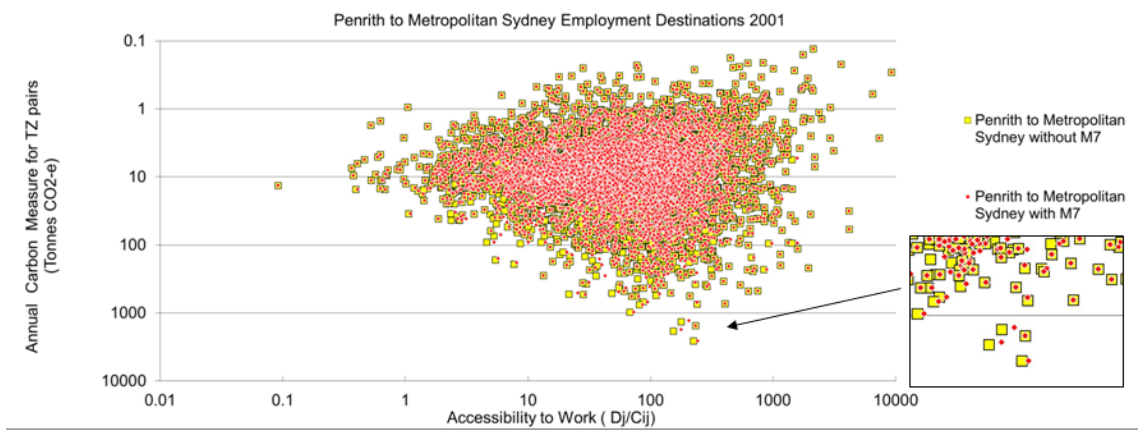

Figure 5: Sustainability performance for Sydney Penrith origins.

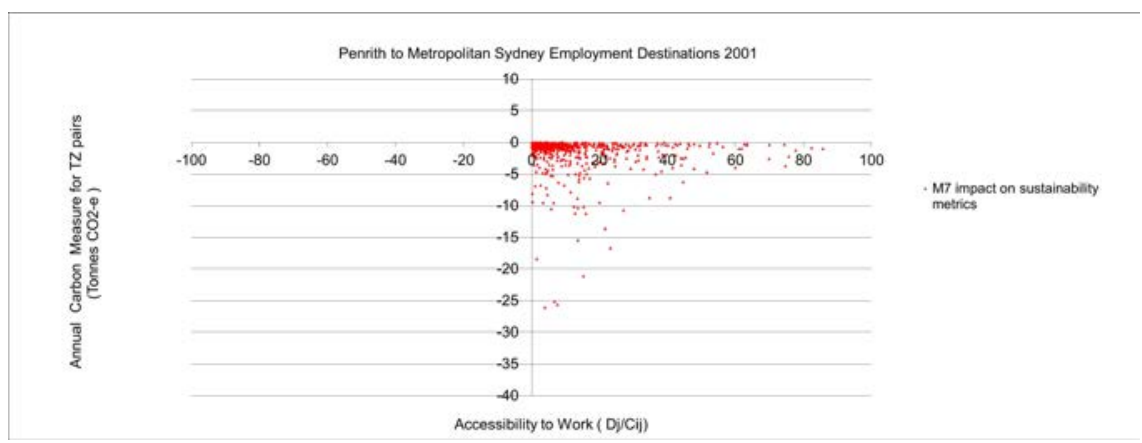

Figure 6: Sustainability performance shift for Sydney Penrith origins.

\section{Conclusions}

\subsection{How effective are these web like spatial transport structures for sustainable citywide accessibility}

This paper has utilised the concept of strategic scans to provide quick turnaround analysis to underpin the assessment of the sustainability performance of spatial 
transport networks across whole cities. The methodology is seen as an important direction in providing tools for testing trunk corridors options in city transport networks. A novel approach to evidence based sustainability metrics as part of the strategic scanning was applied.

The early analysis of the impact of adding an orbital corridor, being examined through the Sydney Case Study, indicates a discernible improvement in the sustainability effectiveness when measured using carbon minimisation and citywide accessibility to jobs. Ongoing case study analysis will be reported in the extended version of this paper in coming months and further extended to include assessment of multimodal orbital links in the coming year.

\subsection{Opportunity and challenges involved in reengineering trunk corridors to multi-modal systems}

The key task for government in the first instance is to identify and reserve suitable corridor easements, with sufficient width to accommodate multi modal transport systems. Sustainability Step 2, not only requires the correct web structure, it also requires the correct mix of system technology, carefully positioned and designed interchanges between the orbital and radial trunk corridors that create the web effect. The trip maker currently uses car for most trips involving longer distances on trunk routes that are not to city centres. The overall car trip time is therefore the baseline to which other system technology has to compare and compete to gain mode share.

A challenge into the future will be changing the paradigm that motorway corridors remain car focused and shift the thinking on repurposing these valuable corridors towards other modes.

\subsection{Continuing work needed and need for collaboration with other researchers}

For the strategic scan approach to provide a more substantial contribution to supporting the sustainability planning decision in cities, a database of metrics applied to multiple cities is needed. This will enable a typology of sustainability performance to emerge over time to guide decision makers on suitable trend breaking urban form and transport network structures tailored to the context of each city.

Ongoing research into the business case modelling and overcoming engineering challenges associated with repurposing motorways for multi modal use, is presenting itself as a parallel area of work needed together with that of sustainability assessment.

\section{Acknowledgements}

Windana Research Pty Ltd and Research Centre for Integrated Transport Innovation (rCITI). To participate in the sustainability database and typology project contact Dr Ken Doust kendoust@unsw.edu.au or windana@maxi.net.au and view our website www.sustainabilitystep2.org 
842 Sustainable Development, Vol. 2

\section{References}

[1] Black, J., Cheung, C., Doust, K., \& Masuya, Y., A Land Use and Transport Scenario Evaluation Model with Optimisation Algorithms and Accessibility and Greenhouse Gas Trade-offs. 12th World Conference on Transport Research, Lisbon, Portugal, 2010.

[2] Doust, K., Coming to a City near You!....Sustainability Step Two. AC21International Forum 2004: Sustainable Transport for Sustainable Cities Satellite Forum, University of Sydney, 2004.

[3] Doust, K., Toward a Typology of Sustainability for Cities. Journal of Traffic and Transportation Engineering (English Edition), 3: 180-195, 2014.

[4] Manning I., The Journey to Work. Urban Research Unit, Australian National University, 1978. 